\section{5}

14

\title{
Search for 4th Generation Quarks with the ATLAS Detector at the LHC
}

\author{
Suyog Shrestha on behalf of the ATLAS Collaboration \\ Iowa State University
}

\begin{abstract}
We present the results from the searches for fourth generation quarks using data samples collected in 2011 by the ATLAS detector at the Large Hadron Collider (LHC) at CERN.
\end{abstract}

Keywords: top quark, 4th generation, new heavy quarks, vector-like quarks

PACS: $14.65 . J k, 14.65 . \mathrm{Ha}$

\section{INTRODUCTION}

A 4th generation of quarks is predicted in a number of theories and stands in agreement with electroweak precision data [1]. Furthermore, CP violation can be significantly enhanced to a level that it might account for the baryon asymmetry in the Universe [1]. At the LHC proton-proton collider, 4th generation quarks can be either produced strongly in pairs or singly via electroweak production. In the following we present searches for pair production of these new heavy quarks performed by the ATLAS detector at the LHC.

\section{SEARCH AND RESULTS}

We performed a search for a pair of $t^{\prime}$ quark, a heavy up-type quark, in the lepton+jets final state [3], characterized by a high transverse momentum $\left(p_{T}\right)$, isolated $e$ or $\mu$, high missing transverse momentum $\left(E_{\mathrm{T}}^{\text {miss }}\right)$, and $\geq 3$ jets. The reconstructed heavy quark mass $\left(m_{\text {reco }}\right)$ is the primary discriminating variable. For events with exactly 3 jets, $m_{\text {reco }}$ is taken to be the invariant mass of the 3 -jet system. For events with $\geq 4$ jets, $m_{\text {reco }}$ is estimated by performing a kinematic likelihood fit. Good agreement between data and background prediction is observed in the $m_{\text {reco }}$ distribution. In the absence of any 
significant data excess, upper limits on $t^{\prime}$ quark pair production cross section are derived using the $C L_{S}$ method (as shown in Fig. 1). At the 95\% confidence level a $t^{\prime}$ quark with mass $<404 \mathrm{GeV}$ that decays entirely via $t^{\prime} \rightarrow W b$ is excluded.
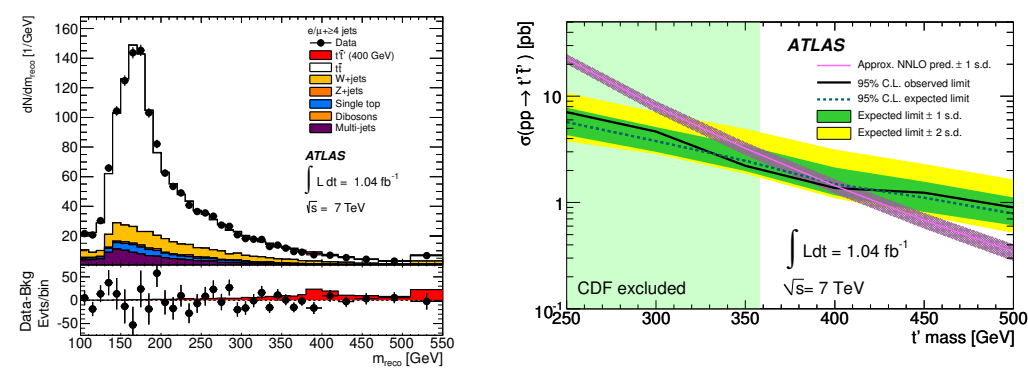

FIGURE 1. (Left): $m_{\text {reco }}$ distribution in the combined $e / \mu+4$ jets channel. (Right): Observed and expected $95 \%$ C.L. upper limits on $t^{\prime}$ quark pair cross section as a function of $t^{\prime}$ quark mass [3].

\section{Search for heavy quark $Q$ pair with subsequent decays $Q \rightarrow W q$}

We performed a search for a pair of heavy quarks $Q \bar{Q}$ with the decay $Q \rightarrow W q$, where $q=u, d, c, s, b$, in the dilepton final state by requiring 2 charged leptons, large $E_{\mathrm{T}}^{\text {miss }}$ and $\geq 2$ jets [4]. $Q$ can be any new heavy quark, though the up-type 4 th generation quarks decaying through weak charged currents are used as a benchmark. The reconstructed mass of the heavy quark ( $\left.m_{\text {Collinear }}\right)$, is the final discriminant. Good agreement between data and background prediction is observed in the $m_{\text {Collinear }}$ distribution. In the absence of any significant data excess, upper limits on the $Q$ pair production cross section are derived using the $C L_{S}$ method (as shown in Fig. 2). At the $95 \%$ confidence level a heavy quark, $Q$, with mass $<350 \mathrm{GeV}$ that decays entirely via $Q \rightarrow W q$ is excluded.
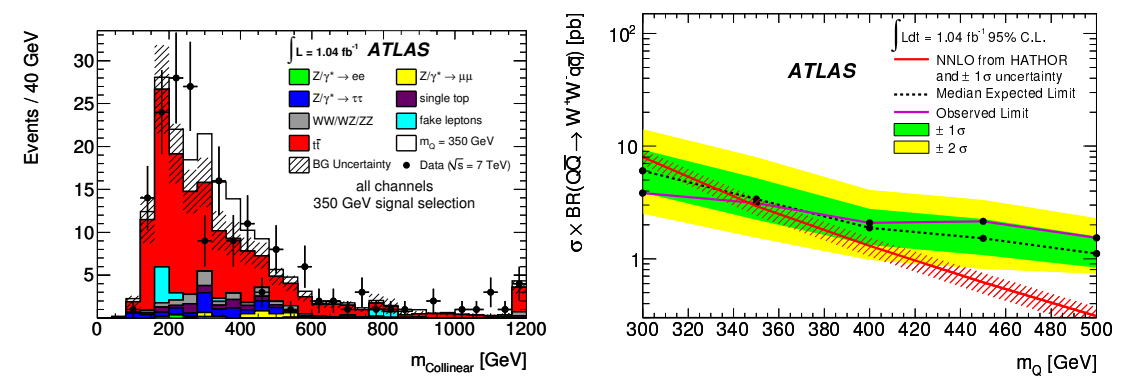

FIGURE 2. (Left): $m_{\text {Collinear }}$ distribution for the sum of $e e, \mu \mu$, and $e / \mu$ channels after applying the final selection for $m_{Q}=350 \mathrm{GeV}$. (Right): Observed and expected 95\% C.L. upper limits on $Q$ pair cross section as a function of $Q$ mass [4].

\section{Search for $b^{\prime}$ quark pair with subsequent decays $b^{\prime} \rightarrow W t$}

We performed a search for a fourth generation down-type quark, $b^{\prime}$, with subsequent decay $b^{\prime} \rightarrow W t \rightarrow W W b$ [5]. Pair production of $b^{\prime}$ quarks leads to $4 \mathrm{~W}$ bosons and 2 $b$ quarks. In the decay mode studied here, one of the $W$ bosons decays leptonically 
and others decay hadronically. The final signature consists of exactly 1 charged lepton, large $E_{\mathrm{T}}^{\mathrm{miss}}$ and $\geq 6$ jets. The distribution of the number of events as a function of jet multiplicity $\left(N_{\text {jets }}=6,7, \geq 8\right)$ with $0,1, \geq 2$ reconstructed $W$ boson is the final discriminant. The number of reconstructed $\mathrm{W}$ bosons $\left(N_{W}\right)$ is defined as the number jet pairs within $\Delta \mathrm{R}<1.0$ and with an invariant mass in the range $70-100 \mathrm{GeV}$. In the absence of any significant data excess, upper limits on the $b^{\prime}$ pair production cross section are derived using the $C L_{S}$ method (as shown in Fig. 3). At the $95 \%$ confidence level a $b^{\prime}$ quark with mass $<480 \mathrm{GeV}$ that decays entirely via $b^{\prime} \rightarrow W t$ is excluded.
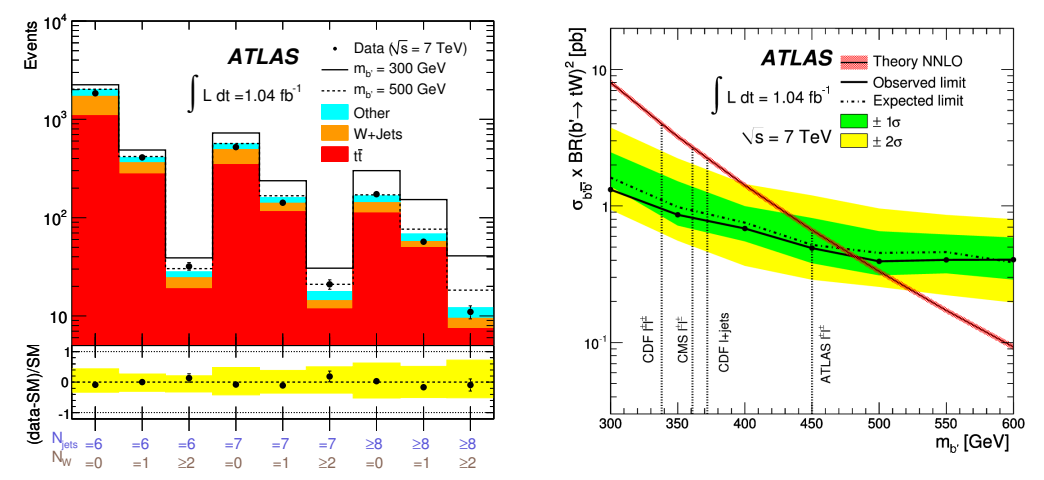

FIGURE 3. (Left): Distribution of the number of events observed for $N_{\text {jets }}=6,7, \geq 8$ with $N_{W}=0,1$, $\geq 2$. (Right): Observed and expected $95 \%$ C.L. upper limits on the $b^{\prime}$ pair cross section as a function of $b^{\prime}$ mass [5].

\section{Search for $b^{\prime}$ pair with subsequent decays $b^{\prime} \rightarrow Z b$}

Using a dataset collected in 2011 with integrated luminosity of $2 \mathrm{fb}^{-1}$, we performed a search for a pair of vector-like $b^{\prime}$ quarks with the subsequent decay $b^{\prime} \rightarrow Z b$ with the $Z$ boson decaying to an electron-positron pair [6]. The mass distribution of large transverse momentum $b^{\prime}, \mathrm{m}(Z b)$, is tested for an excess. In the absence of any significant data excess, upper limits on the $b^{\prime}$ pair production cross section are derived using the $C L_{S}$ method (as shown in Fig. 4). At the 95\% confidence level, a $b^{\prime}$ quark with mass $<400$ $\mathrm{GeV}$ that decays entirely via $b^{\prime} \rightarrow Z b$ is excluded. In the case of a vector-like singlet $b^{\prime}$ quark mixing only with the third generation, masses $m_{b^{\prime}}<358 \mathrm{GeV}$ are excluded.
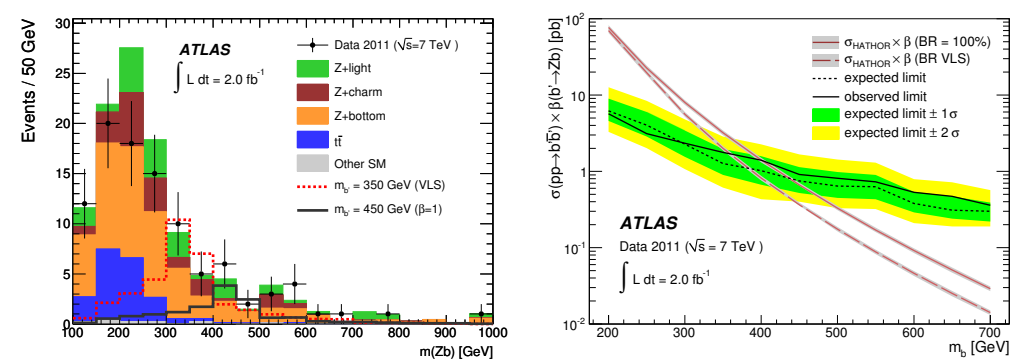

FIGURE 4. (Left): Mass distribution of the $b^{\prime}$ quark candidates. (Right): Observed and expected $95 \%$ C.L. upper limits on $b^{\prime}$ quark pair cross section as a function of the $b^{\prime}$ quark mass [6]. 


\section{ACKNOWLEDGMENTS}

62

63

I would like to thank the ATLAS collaboration for providing me the opportunity to attend the CIPANP 2012 and the Iowa State University High Energy Physics Group for making it financially possible for me to attend the conference.

\section{REFERENCES}

1. See, e.g., B. Holdom, W. S. Hou, T. Hurth, M. L. Mangano, S. Sultansoy, and G. Ünel, and references therein., PMC Physics A 3, 4 (2009) and references therein

2. ATLAS Collaboration, JINST 3 S08003 (2008)

3. ATLAS Collaboration, Phys. Rev. Lett. 108, 261802 (2012)

4. ATLAS Collaboration, Phys.Rev. D86 012007 (2012)

5. ATLAS Collaboration, Phys.Rev.Lett. 109032001 (2012)

6. ATLAS Collaboration, Phys. Rev. Lett. 109, 071801 (2012) 